\title{
Factors Influencing Knowledge and Attitude Among the Caregivers of Patients with Schizophrenia at Mathari Teaching and Referral Hospital, Kenya
}

\author{
Edna Anab ${ }^{1}$, Agatha Onyango ${ }^{2}$, Catherine Mwenda ${ }^{3}$ \\ ${ }^{1}$ Department of Public Health and Community Development, Maseno University, Kisumu, Kenya \\ ${ }^{2}$ Department of Nutrition and Health, Maseno University, Kisumu, Kenya \\ ${ }^{3}$ Department of Medical and Surgical Nursing, Mount Kenya University, Thika, Kenya
}

Email address:

ednanab@yahoo.com (E. Anab), aconyi912@gmail.com (A. Onyango), mutungacs@gmail.com (C. Mwenda)

To cite this article:

Edna Anab, Agatha Onyango, Catherine Mwenda. Factors Influencing Knowledge and Attitude Among the Caregivers of Patients with Schizophrenia at Mathari Teaching and Referral Hospital, Kenya. World Journal of Public Health. Special Issue: Health Dynamics.

Vol. 5, No. 1, 2020, pp. 1-11. doi: 10.11648/j.wjph.20200501.11

Received: October 24, 2019; Accepted: January 6, 2020; Published: January 9, 2020

\begin{abstract}
Schizophrenia is a mental disease that affects how a person feels, behaves and thinks. About 51 million people worldwide suffer from Schizophrenia globally. The main objective of the study was to determine factors that influence the knowledge and attitude of the caregivers of schizophrenic patients at Mathari Teaching and referral Hospital. Caregiver's knowledge facilitates recognition of mental illness and health-seeking behavior. A cross- sectional descriptive study design was carried out to assess factors influencing knowledge and attitude among the caregivers of patients with schizophrenia at Mathari teaching and referral hospital, Kenya. To generate qualitative information, on knowledge and attitude the nurse in charge of the ward was selected as key informants. Data was collected using questionnaire and key informant interview. Qualitative data were analyzed through content analysis, similar themes were grouped. Schizophrenia was diagnosed using diagnostic and statistical manual of mental disorder (DSM-V-TR) criteria. A total of 303 caregivers visiting the patients with schizophrenia in the wards were selected for study using systemic sampling technique. Pearson correlation coefficient, logistic regression and multiple regression was used to determine the magnitude of the correlation between dependent and independent variables. Most (72\%) of the participants did not know the name of the condition which the patient was suffering from. Majority, $164(54.1 \%)$ of the caregivers had a positive attitude towards schizophrenia. There was a perfect positive correlation with the length of time the patient had suffered from the condition (.08423 zero order) with the knowledge of the caregiver. From the study finding, there was a perfect positive correlation with age, gender, highest level of education and nature of occupation $(.06543, .04186, .0088$ and .0039$)$ with the knowledge of the caregiver. There was also a perfect positive correlation with age, gender religion, relationship to client, and nature of occupation $(.0054, .0009 .0357, .0574$ and 0068 zero orders respectively). Study findings indicated a positive correlation between knowledge and the attitude since a caregiver with more knowledge had a positive attitude. The study recommends, treatment literacy classes for caregivers of schizophrenia patients.
\end{abstract}

Keywords: Attitude, Caregivers, Knowledge, Schizophrenia, Mental Health

\section{Introduction}

Schizophrenia is a mental disorder that is characterized by abnormalities in perception or expression of reality. Schizophrenia is a mixture of positive and negative symptoms that present for a significant portion of a month but with continuous signs of disturbance persisting for at least six month [1]. Positive symptoms reflect distortion of normal functions, include delusions and hallucinations. Negative symptoms include, lessening of normal functions such as flattening of affect [2]. There are subtypes of schizophrenia like paranoid, hebephrenic also called disorganized, catatonic, undifferentiated, simple and residual schizophrenia. Schizophrenia typically strikes young people 
at the very time they are establishing their independence and can result in lifelong disability and stigma [3].

About 40 per cent of in-patients in health facilities suffer from mental disorders, according to the Kenya Mental Health Policy 2015-2030. Though mental disorders are common and widespread, a majority of Kenyans who suffer from these illnesses do not get the care and treatment they need. A Kenya National Commission on Human Rights (KNCHR) audit of the mental health system in Kenya estimates that out of six people with a mental illness, five do not receive any treatment. In Kenya, at least one in every four people that present to a healthcare facility do so for treatment of a mental health issue [4]. According to the World Health Organization, Kenya's mental disorders account for $5.9 \%$ of the total global burden. An estimated 90 per cent of the one million suicides globally are associated with some form of mental disorder namely stress, depression, bipolar disorder and schizophrenia [5-7]. Schizophrenia is a more severe form of mental illness but it is also treatable and manageable. In Mathari Hospital once a patient is diagnosed with schizophrenia they are initiated on anti psychotic drugs, offered recreation social therapies and psychosocial counselling.

Schizophrenic patient may not understand they need to seek medical interventions due to the disease process. The patient requires a caregiver to support to keep them oriented. The caregiver's knowledge about schizophrenia may affect the health seeking behavior and response to treatment. The caregiver supports the patient through early recognition of mental illness, seeking health interventions and adhering to treatment [6]. The caregiver's of mentally ill patients tends to be confused or panic in case they do not understand why the person is acting in a strange way. One may not know how to react and what to do during such psychotic episodes. With treatment, persons with schizophrenia can lead pretty normal lives. Untreated, almost all forms of mental illness can lead to severe consequences such as suicide, homicide, homelessness, poverty and permanent psychosis [8]. This study seeks to evaluate the knowledge of the caregivers of schizophrenia patients. The attitude portrayed by the caregivers' shows how eloquent they are with their role. Acceptance makes the patient feel loved by the family and caregivers thus resulting in improved quality of life. Rejection makes the caregiver feel burden of living with a mentally ill relative. Caregivers who are more understanding, encouraging and have a compassionate attitude towards the patient help them to achieve healthier social functioning. Thus, this makes the caregiver able to adjust their reaction according to the patient's emotional state and needs, these acts as a buffer since it protects them from a risky reaction that could trigger a relapse. This study seeks to assess the attitude of the caregivers of Schizophrenia patients.

\subsection{Statement of the Problem}

Schizophrenia causes more lengthy hospitalization, more chaos in family life, more exorbitant costs to individuals and government; and more fears than the other mental health disorders. Schizophrenia from a public health perspective, is a major concern as the onset of illness occurs in the productive age group of $15-35$ years resulting in chronic disability [9]. Schizophrenia is a treatable disorder, treatment being more effective in its initial stages. Relapse in schizophrenia predicts poor prognosis, bring about deterioration in social, occupational and financial status thus increase the burden of care on the family [10]. Comparing with other diseases such as HIV/AIDs there is good retention to care and prognosis when medical interventions are combined with the patient having a treatment supporter such as a well informed caregiver [11].

Inorder for the caregiver to be effective in their role they should be able to understand the causes and management of the condition. The knowledge and attitude of the caregivers boost their capacity which can act as catalysts to reduce some of the symptoms associated with Schizophrenia. The patients are prone to dangerous criminal activities. The caregiver's attitudes could act as a trigger for depressive symptoms, suicidal ideation and substance abuse. Currently in Kenya, limited study has been conducted on knowledge and attitides of the caregivers of schizophrenia.

\subsection{Objectives of the Study}

\subsubsection{General Objective}

To determine factors that influence the knowledge and attitude of the caregivers of schizophrenic patients at Mathari Teaching and referral Hospital.

\subsubsection{Specific Objectives}

1. To assess the caregiver's knowledge on Schizophrenia

2. To assess the caregiver's attitude regarding Schizophrenia

3. To assess the influence of the individual factors on the caregiver's knowledge and attitude regarding Schizophrenia

4. To assess the influence of the caregiver's factors on the caregiver's knowledge and attitude regarding Schizophrenia

5. To establish the relationship between the individual factors and caregiver factors that influence the caregivers' knowledge and attitude of schizophrenia.

\subsection{Research Questions}

1. What knowledge do the caregivers have regarding Schizophrenia?

2. What is the attitude of the caregivers towards Schizophrenia?

3. How does the individual factors influence the caregiver's knowledge and attitude regarding Schizophrenia?

4. How does the caregiver factors influence the caregiver's knowledge and attitude regarding Schizophrenia?

5. Does the individual factors and caregiver factors influence the caregivers' knowledge and attitude regarding Schizophrenia?

\subsection{Significance of the Study}

Given the paucity of research in this area in Kenya, this 
study is important in contributing to information that will help answer critical questions. Furthermore, there is no single study done in Kenya examining the support sytem such as caregivers of the patient diagnosed with schizophrenia. As critical questions on these areas remain unaswered, the present study was significant in examining the biggest mental illness referral and teaching hospital and providing rich data and information regarding caregivers knowledge and attitude about schizophrenia.

These findings are accounts of caregivers and provide insights on opportunities available for caregivers to be better treatment supporters for persons with Schizophrenia. Contributing to the growing body of knowledge regarding the condition makes the caregiver more effective in their role as the patient's support system. These findings are a good opportunity for the formulation of family interventions based on the identified gaps resulting to caregiver's full

\section{Independent variables}

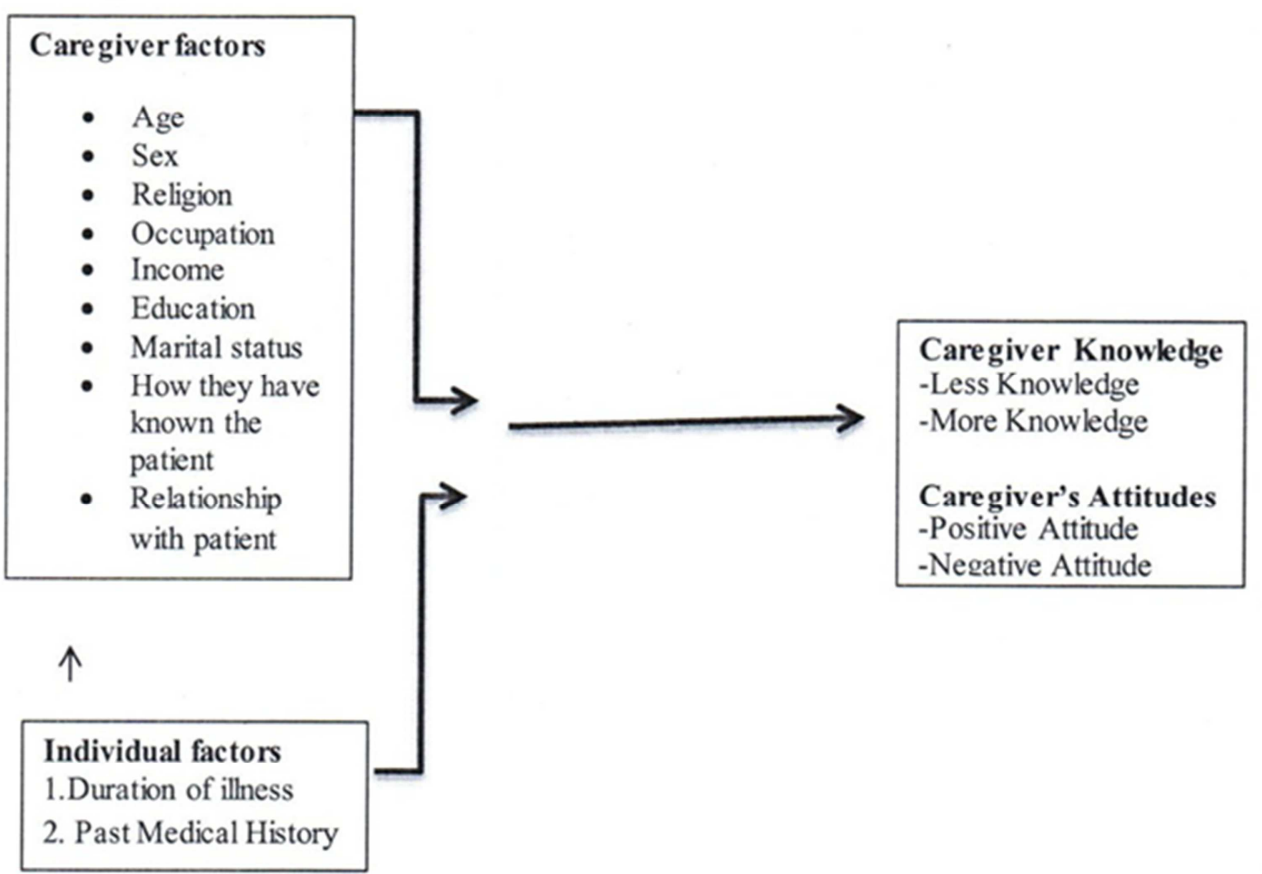

involvement in the care and management of the patient. Also, broaden patient's support system through sensitization and education of the public.

\subsection{Conceptual Framework}

This study was based on the following conceptual framework; the dependent variable was knowledge and attitude while the independent variable constituted of Individual factors (past medical history and duration of illness) and caregiver factors (age, sex, religion, occuppation, income, education attained, marital status and caregiverpatient relationship). The assumptions taken by the researcher were, that the independent variables independently play a role in shaping knowledege and attitude of the caregiver regarding schizophrenia. Figure 1, gives a schematic representation of the model.

\section{Dependent variable / Outcome}

Figure 1. Conceptual framework.

\section{Research Methodology}

This section describes the general methodology used in carrying out this research. It discusses the research design, the population and sample design, the research procedure, the data collection and data analysis methods.

\subsection{Study Setting}

Mathari Teaching and Referral Hospital is a National Referral and Teaching Hospital situated along Thika road opposite Muthaiga Police Station about five kilometers from the Nairobi city center. The hospital is divided into two sections: a civil section for ordinary patients and a maximum-security unit for mentally disorderly offenders referred for assessment and treatment by the criminal justice system.

\subsection{Study Population}

The study population were caregivers aged eighteen years and above who was a caregiver of a patient diagnosed with schizophrenia using DSM-V-TR admitted in Mathari Hospital (Schizophrenic patient who was selected using systematic sampling). The study targeted a total of 303 caregivers. 


\subsubsection{Inclusion Criteria}

A caregiver of a schizophrenic patient admitted at Mathari Teaching and Referral Hospital and who is a consenting adultaged 18 years and above.

\subsubsection{Exclusion Criteria}

A caregiver of a patient diagnised with other psychiatric and mental health disoders based on DSM-IV diagnosis. Caregivers who are not willing to participate in the study.

\subsection{Research Design}

This study adopted a descriptive cross-sectional mixed method utilizing both quantitative and qualitative were data was collected once and analyzed.

\subsection{Sample Size Determination and Sampling Procedure}

\subsubsection{Sample Size Determination}

The sample size was calculated using the Fisher sample size calculation standard formula.

Sample formula $\mathrm{n}$ study population was less than 10,000

$$
\text { Therefore, } \mathrm{nf}=\mathrm{n} \div 1+(\mathrm{n} / \mathrm{N})
$$

Where

$\mathrm{nf}=$ the desired sample size when the population is less than 10,000

$\mathrm{n}=$ the desired sample size when the population is more than 10,000

$\mathrm{N}=$ the estimated population size

Therefore; $\mathrm{nf}=384 /(1+(384 \div 975)$

$=275$ respondents

Plus $10 \%$ for non-responses

: $275 \times 10 / 100=27.5$ Thus; 28 respondents

The final sample size was $275+28=303$ respondents of the schizophrenic patients.

\subsubsection{Sampling Procedure}

Systematic sampling is a type of probability sampling method involving the selection of elements from an ordered sampling frame. Systematic sampling technique was used to select nth patients' family members or significant others. The nth patient diagnosed with schizophrenia admitted in Mathari Hospital after confirming the diagnosis by reviewing patient's records. Selection was done as follows:

Stage 1: Selection of hospital

Mathari Teaching and Referral Hospital was selected purposively, since it is a National hospital receiving mentally ill patients' country wide where the highest numbers of schizophrenic patients are found.

Stage 2: Selection of the wards

Male and female civil wards were selected purposively because they admit mentally ill patients daily.

Stage 3: Selection of Schizophrenic patients

A list of the names of the patients was generated from the inpatient register from all the seven wards as the sampling frame. Using a skip pattern, every nth patient on the list was selected, where $\mathrm{n}$ was the sampling interval, calculated by dividing the total number of respondent's /sampling frame with the sample size $\left(\mathrm{n}=3^{\text {rd }}\right.$ i.e. $\left.975 / 303\right)$.

Stage 4: Selection of Caregivers

All the caregivers of the nth patient had an equal chance of participating in the study. To ensure total representation of the entire population, the caregivers were drawn from all the seven wards in the hospital. Data was collected in all selected wards until the required number of participants was attained.

Stage 5: Selection of Key Informants

The Nurse Incharge of the nine wards were selected as the key informants since they could be able to give information on the management of schizophrenia.

\subsection{Data Collection Instruments}

\subsubsection{Questionnaire}

A questionnaire was used to yield information on knowledge and attitude of the caregiver regarding the care of Schizophrenia. Questionnaires were also used to collect qualitative data. The questions comprised of the following parts which was completed by the researcher and research assistances as they interviewed the respondents: Sociodemographic information about the caregiver, Caregivers' knowledge about Schizophrenia and Caregivers' attitude about Schizophrenia

\subsubsection{Key Informant Interview}

The key informant interview was used to collect qualitative data. This yielded information on the role of the caregivers of the Schizophrenic patients.

One section of the guide solicited general information and the other remaining section was based on the role of the caregivers.

\subsection{Pre-testing}

Pre-testing was done on 28 respondents that is $10 \%$ of the sample size. Pilot was conducted at Mathari Hospital inpatient ward and those who were included in the piloting of the instrument were excluded from the sample size. During the pre- testing the researcher assessed the clarity of the instrument and the ease of the use of the instrument. This process checked whether all the questions asked were eloquent and had the same meaning to all respondents. This process also tested the rate of response depending on the type of response. Following this exercise, some revisions on the tools were made. This data was used to refine the questionnaire and key informant guide that was finally administered during the research process.

A key deliverable from this process was training of research assistants on the objectives of the study and how-to best approach data collection in order to reduce variability and possible distortion of information during questionnaire administration.

\subsection{Data Collection Procedures}

\subsubsection{Questionnaire}

The questionnaires were researcher administered 
questionnaire, where the researcher asked the question directly to the respondent and in certain circumstances translated the questionnaire to the respondents. Assistance was sorted from the nurses who could translate the questions in various native languages in case of language barrier.

The Questionnaire was also translated to Kiswahili. The researcher confirmed the diagnosis of the patient by review of patients' records that indicate the diagnosis of the patient according to the psychiatrist.

A caregiver was categorized as having adequate knowledge if they were able to identify the name of the condition, how long the patient has suffered from the condition, the cause of the condition, manifestation of the condition, what aggravates the condition and if the patient was on any prescribed medication before being admitted. If a caregiver was able to answer at least six of the above questions they were considered adequate knowledge and if not able to answer the above six questions they were considered as having inadequate knowledge.

Information on attitude was evaluated through likert scale, attitudinal statements was utilized to capture the attitude of respondents on schizophrenia. This was after rating on the scale coded to depict statements of agreement, not know and disagreement as positive, neutral and negative respectively. In consolidated questionnaire there is section on caregiver's socio dermographic information, knowledge and attitude. The questionnaire was written in both English and Kiswahili languages to allow easy interpretation by the correspondents. The process of filing the questionnaire was conducted at the wards while the respondents were visiting the patients, each data collection session took about 30 minutes.

The questionnaire was administered by the researcher or trained research assistance. Once the raw data obtained from the field was edited, coded and entered into SPSS and analyzed.

\subsubsection{Key Informant Interview}

Nurse Incharge of the nine psychiatric wards of Mathari Teaching and Referral Hospital, were selected as the key informants.

Ultimately, nine nurse incharge were interviewed. Each interview took approximately 20 minutes. The tool was in English and contained seven key questions. The key informant interview tool was generated to find out the involvement of the caregiver of Schizophrenia patients on the care and management of the condition.

\subsection{Validity and Reliability}

Validity was done using content validity whereby the tool was reviewed by an expert in the subject area and my supervisors. Reliability was checked by analyzing the data obtained from the 28 respondents who took part in the pilot study. Using the Cronbach's alpha or coefficient alpha which the questionnaire had 34 items, the data was then analyzed yielding Cronbach value of 0.732 which falls within the acceptable standard alpha range value of $0.8>\mathrm{a} \geq 0.7$ an indicator that the data collection instrument was reliable.

\subsection{Measurement of Variables}

\subsubsection{Dependent Variable}

The dependent variables for this study were knowledge of the caregivers on schizophrenia and their attitude towards the illness.

i. Knowledge

A caregiver can be categorized as having either adequate knowledge or inadequate knowledge. A caregiver can be categorized having adequate knowledge if they are able to identify the name of the condition, how long the patient has suffered from the condition, the cause of the condition, manifestation of the condition and what aggravates the condition.

ii. Attitude

A caregiver can be categorized as having either positive attitude or negative attitude.

\subsubsection{Independent Variables}

The independent variables for this study were characterized as summarized below:

i. Individual Factors

This are the patient's individual factors such as length of time the patient has been suffering from Schizophrenia and past medical history.

ii. Length of Time the Patient has been suffering from Schizophrenia

This was defined in either months or years the patient had suffered from Schizophrenia. The assumption at the time before data collection was that duration of illness could possibly influence the caregivers' knowledge and attitude.

iii. Past Medical History

The study also sought out historical instances of recurrence or relapse of the condition in patients enrolled in the study.

\subsubsection{Caregiver Demographic Characteristics}

The secomprisedof data and information on age, sex, educational level, occupation and marital status. Age was defined as years since birth; sex as male or female; occupation as the nature of income generation whether formal or informal, education level as highest knowledge level of education attained. The solicited data facilitated assessment of the characteristics of the caregivers as factors that influenced knowledge and attitude.

\subsection{Data Analysis}

\subsubsection{Qualitative Data Analysis}

Qualitative data were analyzed using content analysis with a focus on themes. After transcription of data, a critical review process looking at categories and creating broad themes and concepts leading to the analysis of the data was carried out.

In summary, the raw data was subjected to the following processes:

1. Transcribed data were reduced through the process of critical reading and subsequent categorization of materials based on the study objective.

2. The open-ended response were then organized 
according to the research objective and themes

3. Data was then interpreted. This involved making decisions and drawing conclusions related to the evaluation questions and objectives. During this process, the organized data was explored in-depth.

\subsubsection{Quantitative Data Analysis}

Data was sorted out, cleaned, categorized, coded and entered into SPSS version 20.0.0 software then analyzed using descriptive statistics to generate frequency tables and charts. Further to this, to understand relationship between variables, regression analysis was used to determine coefficients. Additionally, this was also to facilitate understanding whether the dependent variables which are knowledge and attitude indeed depended on the independent variables which are individual factors and caregiver's factors. Linear regression and multi regression were then carried out to determine the magnitude and linearity of correlation between the factors to predict the association between the attitude and knowledge. For all analyses, zero order was interpreted as -1.0 indicate perfect negative correlation, 1.0 perfect positive correlation and 0 no linear relationship between the variables. Variance Inflation Factor (VIF) interpreted as 1 not correlated, 1-5 moderately correlated and $<5$ highly correlated.

Multi regression y is interpreted as-1.0 indicate perfect correlation, 1.0 positive correlation and 0 no relationship between the variables. The y value was be used to gauge the magnitude of influence of the independent variables and thus their influence on the dependent variables which are knowledge and attitude.

\subsection{Ethical Considerations}

Authority to carry out the study was sought from, the Medical Superintend of Mathari Teaching and Referral Hospital gave institutional permission to conduct research. The researcher obtained permission from the Kenyatta National Hospital/ University of Nairobi- Ethical Research Commission (KNH/UoN- ERC) reference number $\mathrm{KNH}-$ $\mathrm{ERC} / \mathrm{A} / 485$. For the University institutional level, the researcher obtained permission from the School of Graduate Studies, Maseno University. The purpose of the study was fully explained to the study subjects and written informed consent obtained before inclusion in the study. The consent note was also translated in Kiswahili language.

It was explained that participation in the study was voluntary and that there would be no material gain on participation. Participants were under no obligations to answer questions they were not comfortable with.

The study subjects were assured confidentiality. It was explained to them that their names would not be used and the information cannot be traced back to them. They were informed that the study would be non-invasive and therefore there would be no physical or psychological harm.

It was very clear that their participation was a key step in generating knowledge to further the research agenda and ultimately focus and improve on prognosis and management of Schizophrenia.

In line with the principle of beneficence, caregivers who expressed burn out were referred to the nurses for psychotherapy. The researcher helped particular caregivers to plan how they could further their knowledge on the condition.

\section{Results}

In summary a total of 303 caregivers and 9 key informants were interviewed. The 303 participants were caregivers of patients who were diagnosed with schizophrenia they were approached while visiting the Schizophrenia patient. The 9 key informants were the nurses working at Mathari Hospital. The study had $100 \%$ response rate without any drop out.

\subsection{Socio-Demographic Characteristics of the Respondents}

Most, 191 (63\%) of the respondents were female while 112 $(37 \%)$ were male. With regard to County of residence, 153 $(51 \%)$ were from other counties while $6(2 \%)$ respondents came from Uganda. A good number, 110 (36.3\%) of the caregivers were aged between 35-44 years while 40 (13.1\%) were aged either 45 years or above. Most of the participants $88.9 \%$ were Christians while $6.1 \%$ Muslim. In the question to sort level of education most, $156(51.1 \%)$ of the respondents have attained secondary school level while $12(4.0 \%)$ stated that they had no formal education.

Most, of the respondents $153(50.5 \%)$ have informal business while $40(13.1 \%)$ stated they were not employed. Majority of the caregiver's $286(94.4 \%)$ of the caregivers were related while $17(5.6 \%)$ were friends of the patient. With regard to how long the caregiver had known the patient most, $199(65.7 \%)$ had known the patient for above 10 years while only $6(5.3 \%)$ had the patient for 6-11 months.

From the key informant's interview most of the patients admitted in Mathari Hospital wards are schizophrenia patients. All the incharges mentioned involuntary as the leading type of admission. Involuntary admissions is whereby the patient is brought in facility against their will due to having psychotic episodes.

\subsection{Knowledge of the Caregivers of Schizophrenic Patients Regarding Schizophrenia}

\subsubsection{Response of the Respondent on the Name of the Condition}

Majority, $219(72 \%)$ of the caregiverswere not aware of the nameof the mental condition the patient was suffering from only 84 (28\%) knew the name of the condition.

\subsubsection{Period the Patient had Suffered from the Condition}

It is evident that most, $192(63.4 \%)$ of the patients had suffered from Schizophrenia for a period of three years and above while 40 (13.2\%) had suffered from Schizophrenia for a period of one to two years as illustrated in Figure 2 below. 


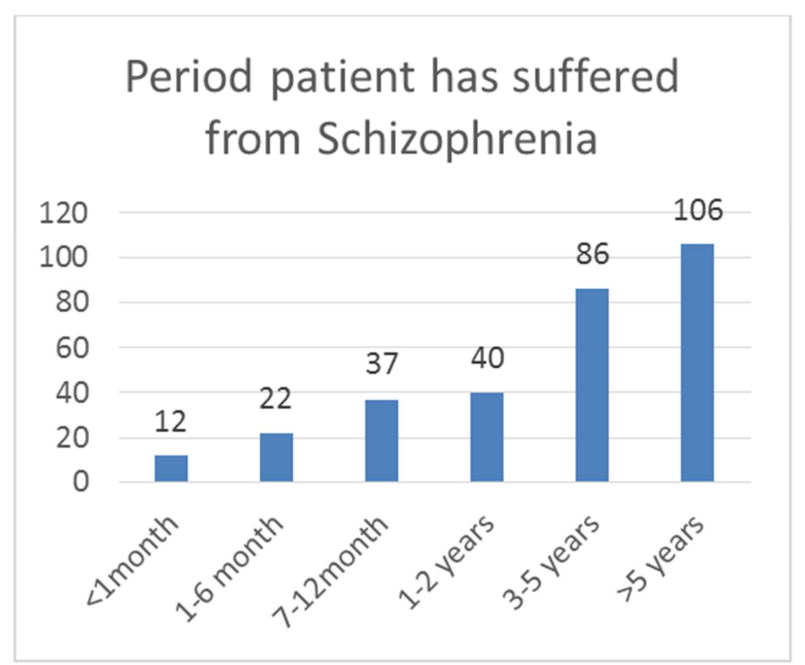

Figure 2. Period patient has suffered from the condition.

\subsubsection{Causes of Schizophrenia}

The caregivers attributed stress $128(42.2 \%)$ the leading cause of Schizophrenia. Other additional causes which was likely to cause Schizophrenia was death of a family member $10(3.3 \%)$ and other illness $4(3.3 \%)$ as illustrated in Table 1 below.

Table 1. Response of the Respondents on Causes of Schizophrenia.

\begin{tabular}{lll}
\hline Causes of Schizophrenia & $\mathbf{n}$ & $\mathbf{\%}$ \\
\hline Stress & 128 & 42.2 \\
Drug/Substance abuse & 74 & 24.4 \\
Hereditary condition & 46 & 15.2 \\
Drug/Substance abuse and stress & 21 & 6.9 \\
Hereditary condition and curse & 18 & 5.9 \\
Cerebral malaria & 14 & 4.6 \\
Death of a family member and stress & 10 & 3.3 \\
Head injury, traumatic events and stress & 10 & 3.3 \\
Head injury & 7 & 2.3 \\
Other illness & 4 & 1.3 \\
Curse & 3 & 1.0 \\
\hline
\end{tabular}

\subsubsection{Manifestation of Schizophrenia}

Most of the caregivers relate the manifestation of schizophrenia as disorganized behavior 196 (64.7\%) while disorganized speech and loss of interest with surrounding accounted for only $4(1.32 \%)$. In addition, other manifestations of Schizophrenia the caregivers could relate the condition to are: problems with memory and thinking 46 (15.2\%), extreme mood change $15(5.0 \%)$, suicidal thoughts $12(4.0 \%)$ and difficulty functioning at school or work accounted for $9(3.0 \%)$.

\subsubsection{Factors That Aggravates Schizophrenia}

The caregivers attribute schizophrenia is mostly aggravated by not using prescribed medications properly 118 (39.0\%) Other factors listed by caregivers such as fatigue, hunger, disturbance, loss of job and anger 15 (5.0\%) also aggravate Schizophrenia.

\subsubsection{Side Effects the Patients Complained After Taking the Drugs}

Out of the 303 caregivers, 214 caregivers indicated that the patient had been on prescribed medication before being admitted. The most common side effects of the drugs by the caregivers are tremors $91(42.5 \%)$ while impaired vision 1 $(0.5 \%)$ was the least raised complained. In addition to the list, complains raised by the patient after taking the drugs ranged from diarrhea, being quiet and confused 36 (16.8\%).

\subsubsection{Response of the Respondent on the Patient Suffered from Any Medical Condition}

Most 240 (79.2\%) of the patients had never suffered from any medical condition while only $63(20.8 \%)$ had suffered from another medical condition.

From the key informants interview apart from being administered antipsychotic drugs the schizophrenia patient also undergoes psychological and social therapies. Psychosocial and social therapy focuses on the patient's relationship with others and the patient participating in recreation activities. The caregivers are educated when the patient is admitted and during the subsequent visit on the management of the condition and prognosis of the patient by the health care workers.

\subsection{Attitude of the Caregivers of the Schizophrenic Patients Regarding Schizophrenia}

\subsubsection{Attitude of Caregiver's Towards Patients with Schizophrenia}

Majority, $164(54.1 \%)$ of the caregivers had a positive attitude, $111(36.6 \%)$ had a negative attitude while $28(9.2 \%)$ had a neutral attitudeas ilustrated in Table 2 below.

Table 2. Attitude of the Caregiver's towards Schizophrenia.

\begin{tabular}{lll}
\hline Attitude of the caregivers & F & \% \\
\hline Positive & 164 & 54.1 \\
Negative & 111 & 36.6 \\
Neutral & 28 & 9.2 \\
\hline
\end{tabular}

\subsubsection{Influence of the Caregiver's Attitude}

There was a perfect positive correlation since the caregivers enjoy being with patient at home, they don't like to talk with anybody about the things that happen to the patient and theydon't like to talk with anybody about the things that happen to the patient $(.0201, .022$ and .670 zero orders respectively). This further depicted by the VIF values that indicate there was moderate correlation among the variable (1.196, 1.346 and 1.317 VIF values respectively). There perfect negative correlation which indicates the caregivers had a positive attitude since they disagree that schizophrenia patient should always be hospitalized and they prefer to hinder the illness (-.1777 and -.069 zero order). From both VIF and zero order it shows there was positive attitude towards schizoprenia

\subsubsection{Perception of Patient's Current Condition}

Most, 257 (84.8\%) of the caregivers were satisfied with the current condition of the patient while $46(15.2 \%)$ rated the patient's current condition being poor compared to when they were first admitted. 


\subsubsection{Perception on Recovery from Schizophrenia}

Majority, $162(53 \%)$ of the caregivers felt the patient will be able to recover completely while 141 (47\%) felt they will not recover from the condition.

\subsection{Influence of the Individual Factors on the Caregiver's Knowledge and Attitude Regarding Schizophrenia}

\subsubsection{Influence of Individual Factors on Caregiver's attitude Regarding Schizophrenia}

There was a perfect negative correlation since the caregivers do not prefer to hide the illness from the rest of their family and friends, unwillingness to talk with anybody about the things that happen to their relatives and assertion that patients with schizophrenia should always be hospitalized (-1.2885, 2.408 and -.9444 zero orders respectively).

This shows the duration the patient had been ill and past medical history does not influence the caregiver's believe of the patient being hospitalized, hide the condition and talk about the condition. The positive correlation shows the individual factors influence the caregiver's attitude of staying with the patient at home and talking about the condition (.062 and .036 zero order). This further depicted by the corresponding VIF values that indicate existence of moderate correlation among the variables $(1.377,1.376$ and $1.141 \mathrm{VIF}$ values respectively.

From the key informants interview most of the caregivers once the patient is newly diagnosed they are really eager to understand the condition. Caregivers who have patients who have suffered for a long time and tend not to improve have a positive attitude.

\subsubsection{Influence of the Individual Factors on Caregivers' KnowledgeRegarding Schizophrenia}

There was a perfect positive correlation with the length of time the patient had suffered from the condition (.08423 zero order) while there was a negative correlation with the patient having suffered from any medical condition before. This shows the period the patient had been ill influence the knowlegde of the caregiver.

From the key informants' interview most of the nurses reported the nurses reported that the clients whose patient had suffered from schizophrenia for long are more knowledgeable on the condition. Most of the respondents said that they knew the illness from the patient's experience, some from the health workers and other through friends. There is high level of involvement by the caregivers of the newly diagnosed patients compared to the ones who had suffered from schizophrenia for a long time. Some of the caregivers of the patients who had incurred relapse had abandoned the patients at the hospital.

\subsection{Influence of the Caregiver's Factors on Caregiver's Attitude Regarding Schizophrenia}

There was a perfect positive correlation with age, gender religion, relationship to client, and nature of occupation $(.0054, .0009 .0357, .0574$ and 0068 zero orders respectively). This was further depicted by VIF that there is moderate correlation between the variables.

\subsection{The Relationship between the Individual Factors and Caregiver Factors That Influence the Caregivers' Knowledge and Attitude of Schizophrenia}

\subsubsection{Relationship between Caregiver' Knowledge and Attitude}

There was a positive correlation between knowledge and the attitude since a caregiver with more knowledge enjoys being with the patient at home, does not believe a schizophrenia patient should always be hospitalized, does not prefer to hide the illness from the rest of their family membes and friend and is okay to talk to other people about the condition (zero order. 0482).

\subsubsection{Further Correlation on Relationship Between the Individual Factors and Caregivers Factor that Influence Knowledge and Attitude}

The results below were modeled from multiple regression to confound the extend to which caregiver's factor and individual factor's influence knowledge and attitude..

There was a negative correlation $(-0.7169)$, the fact that the multiple regressions equation shows that the individual factors that not have a effect on the caregiver factors influence on the knowledge and attitude of the caregivers.

The $y$ value was be used to gauge the magnitude of influence of the independent variables and thus their influence on the dependent variables which are knowledge and attitude.

They are moving to the opposite direction by -0.7169 , as the impact increases by -0.7169 the influence also increases on the other variable.

A Schizophrenia patient can be managed at home and the caregiver should ensure that the patient is brought to facility during clinical appointments. The major challenges that hinder the caregivers from bringing the patient for clinics consultation include financial constraints, lack of transport, refusal by patients and some forget the return date. Other problems mentioned were having stock out of antipsychotic drugs at home and aggressive behaviors of exhibited by the patients. The caregivers expressed both emotional burnout and financial constraints due to taking care of the patient. Most of the respondents expressed feelings of being alone, isolated and labeled due to having a mentally challenged relative.

\section{Discussion}

\subsection{Knowledge of the Caregivers of Schizophrenic Patients Regarding Schizophrenia}

Most, $219(72 \%)$ of the caregivers were not aware of the name of the mental condition the patient was suffering from. This finding is similar to the findings conducted by Magaru in hospital based in India during outpatient clinics, where the term schizophrenia was new to the caregivers but the understanding of the nature of the illness from the caregivers' 
perspective was not restricted to knowing the name of the diagnosis but to general mental illness.

Majority, 192 (63.4\%) of the patients had suffered from Schizophrenia for a period of three years and above this corresponds with the Townsend that schizophrenia is a chronic illness [2].

The major cause of Schizophrenia was stress 128 (42.4\%) while curse $3(1.0 \%)$ was the least attributed cause of Schizophrenia. This findings wereslightly similar to that found in a community based study in Kenya [12] where 44\% psychoactive substances, $18 \%$ inheritance, $15 \%$ life events, $14 \%$ trauma and $9 \%$ witchcraftattributed to cause mental illness. The findings of this two studies contrast to a study conducted in Nigeria, where most $(72.0 \%)$ of caregivers endorsed supernatural causes as most important in the etiology of schizophrenia, while $28.0 \%$ endorsed natural causes. The most frequently endorsed causation was Satan's work $(59.5 \%)$ followed by brain injury $(52.5 \%)$, and curses from enemies (45.5\%) [13]. Based on the findings of various studies conducted in Malaysia and Arabian community also contrast with this study where most of the caregiver's believed schizoprenia is as a result of curse [14]. The belief about mental illness caused by the supernatural power makes the relatives to take mentally ill patients to traditional healers. This may lead to delays in seeking treatment and can lead to poor drug compliance.

Most of the caregivers relate the manifestation of schizophrenia was disorganized behavior 196 (64.7\%), hallucinations $111(36.6 \%)$, loss of interest with surroundings $68(22.4 \%)$, problems with memory and thinking $46(15.2 \%)$ and extreme mood change $15(5.0 \%)$. The caregiver's were able to recognize positive and negative signs/manifestation of schizophrenia inline with DSM V criteria.

Most, 118 (39.0\%) of the caregivers attribute schizophrenia was aggravated by not using prescribed medications properly. This findings supports Gathaiya study conducted in Mathari Hospital, who reported that the major reason for relapse in schizophrenia patients is noncompliance with medication regimen [15]. Failure to comply with medicine regimen can lead to several readmissions, as indicated in the findings from key informant interviews that most of the patient admitted in the wards in Mathari hospital suffer from schizophrenia.

\subsection{Attitude of the Caregivers of the Schizophrenic Patients Regarding Schizophrenia}

Majority, $164(54.1 \%)$ of the caregivers had a positive attitude towards schizophrenia, this is in contrast to a study conducted in Canada-based study examined community attitude towards people with Schizophrenia were the participants had negative attitudes and feelings of social distance toward people with Schizophrenia [9].

There was perfect negative correlation, this shows that most of the respondents had a positive attitude since they believe that a schizophrenic patient should not always be hospitalized and they do not prefer to hide the illness from the rest of the family members and friends (-.177 and -.069 zero orders respectively). The caregivers positive attitude was further depicted since, as the caregivers enjoy being with the patient at home (.0201 zero order).

The most held negative opinion was the caregiver don't like to talk with anybody about the things that happen to patient and they don't like to talk with anybody about the things that happen to patient (.022 and .670 zero orders respectively). This is slightly similar to a community based study were the most widely held negative opinions were that mentally ill people are dangerous to others $(60 \%$ of the respondents), $58 \%$ of the respondents thought that such people are hard to talk to while $56 \%$ thought they are unpredictable [12]. Comparing this study with the study conducted in India, it's in contrast with the findings since, $36 \%$ of the caregivers of schizophrenia patients were comfortable discussing about the illness with others [16].

Most, 257 (84.8\%) of the caregivers were satisfied with the current condition of the patient compared to when they were first admitted this findings contrast with a study conductedin London, were most of the caregivers believed that the patient will improve but they were uncertain if the patient would fully recover or there would be a relapse after recovery. The results of a review study conducted in Japan, the majority felt that one cannot recover from mental illness. Most of the respondents were sure that the illness would be chronic or eventually deteriorate. Despite that, they were convinced that the course of the illness could be influenced by psychiatric treatment as well as by the patients themselves. The assessment of the prognosis of the schizophrenic illness by most relatives appears quite realistic [12].

Majority, $162(53 \%)$ of the caregivers felt the patient will be able to recover completely this is in contrast to a community based study conducted in Kenya, were $47 \%$ thought that the mentally ill patients would never recover even if medically treated [12].

\subsection{Influence of the Individual Factors on the Caregiver's Knowledge and Attitude Regarding Schizophrenia}

The duration the patient has been ill influence the attitude of the caregiver. Most of the respondents had a positive attitude since they believe that a schizophrenic patient should not always be hospitalized, they were okay with talking with other people about what had happened to the patient, they enjoy being with the patient at home and they do not prefer to hide the illness from the rest of their family members and friends $(-1.2885,-2.408, .0201$ and -.9444 zero orders respectively). The more the caregiver understands the patient's medical history the higher the chances of developing a positive attitude. In a study conducted in Nigeria by Oye, on the effect of relapse on the relatives understanding on schizophrenia concurs with the findings of this study since it was found that the relatives who's loved ones had experienced the condition more than one year had a greater understanding of schizophrenia in terms of cause, management and prognosis $[2,6,17]$. The length of time the caregivers have spent, in terms of everyday management of 
the patient, plays a crucial role in the depth at what the caregiver will know. This findings arealso similar to a study conducted by Mbugua, which showed that those who had lived in the same household with a mentally ill person were less likely to hold a negative opinion about them. There was a perfect negative correlation with the patient having suffered from another medical condition before (-.09017 zero order) while there is a perfect positive correlation with the length of time the patient has suffered from the condition (.08423 zero order). The longer the patient had suffered from the condition the more informed the caregiver is in regard to what aggravates the condition, the year the condition started, how the condition manifest and side effects of the drugs.

\subsection{Influence of the Caregiver's Factors on the Caregiver's Knowledge and Attitude Regarding Schizophrenia}

In the study the caregiver's factors was the caregiver socio demographic characteristics. This was assessed to find out if the caregiver's factors influence the caregiver's knowledge and attitude regarding schizophrenia.

There was a perfect negative correlation with marital status, religion, relationship to client and for how long have you lived with/ known the patient (-.0236, -.0219, and -.0146 zero orders respectively). There was perfect positive correlation with age, gender, highest level of education and nature of occupation $(.06543, .04186, .0088$ and .0039). This was further depicted by VIF that there is moderate correlation between the variables. Positive correlation of age, gender and highest level of education is in tandem with results from demographic data since most, 191 (63\%) of the caregivers were female, $110(36.3 \%)$ were aged between 35 44 years and most 260 (85.8\%) had undergone formal education. This indicates that the age, gender and education level influence the caregiver's understanding of the cause, manifestation, factor that aggravates the condition and side effect of the drug. This findings are similar to a community based study [12] were chi square method was used to establish the significance between age with cause and management of mental illness, where the respondents who were more than 60 years were more likely to believe that mentally ill patients are pocessessed by demons and the difference between the age groups was statistically significant $(\mathrm{p}=0.001)$.

There was perfect negative correlation with marital status, highest level of education, and for how long have you lived with/ known the patient (-.0514, -.0544 and -.0349 zero orders respectively). This shows that marital status, highest level of education, and for how long have you lived with/ known the patient did not influence the attitude of the caregiver. This is in contrast with a community study by Mbugua, since negative opinion on whether mentally ill patients would improve with treatment was higher among those without formal education and the difference was statistically significant $(X=29.3, p=.000)$. There was a perfect positive correlation with age, gender religion, relationship to client, and nature of occupation $(.0054, .0009 .0357, .0574$ and 0068 zero orders respectively).
This was further depicted by VIF that there is moderate correlation between the variables. Positive correlation of gender, age, religion, relationship to client, and nature of occupation is in tandem with results from demographic data since most, $191(63 \%)$ of the caregivers were female, 110 $(36.3 \%)$ were aged between $35-44$ years and most 260 (85.8\%) had undergone formal education. Most, 288 (95.0\%) caregivers were either Muslims or Christians this makes them to have a positive attitude since they avoid false belief and they do not believe in curse 3 (1.0\%). Majority, 297 (95.4\%) of the clients were closely related to the patient and had taken care of the client for above 3 years 192 (63.4\%). This indicates that the gender, age, religion, relationship to client, and nature of occupation influence the atittude of the caregiver.

\subsection{Influence the Caregivers' Knowledge and Attitude of Schizophrenia}

There was a positive correlation between knowledge and the attitude since a caregiver with more knowledge enjoys being with the patient at home, does not believe a schizophrenia patient should be hospitalized, does not prefer to hide the illness from the rest of their family members/ friends and is okay to talk to other people about the condition (zero order. 0482).

There was a negative correlation $(-0.7169)$, the fact that the multiple regressions equation shows that the individual factors do not have an effect on the caregiver factors influence on the knowledge and attitude of the caregivers. Knowledge and attitude are moving to the opposite direction by -0.7169 , as the impact increases by -0.7169 the influence also increases on the other variable. Although this results indicate negative relationship between the individual factors and caregivers' factor influence on knowledge and attitude, the positive relationship exhibited before could have emanated from moderate multi linearity among the variables as exhibited by the variance inflation factors.

\section{Recommendations}

1) Treatment literacy classes for caregivers of schizophrenia patients.

2) To foster positive attitude among the caregiver's frequent assessment of their attitude and reassurance about the condition of the patient should be done by the health care workers.

3) The length the caregiver spends with the patient affects the knowledge and attitude, there is need to develop creative strategies to encourage the caregivers to interact with the patients frequently. Strategies such as phone call/ text reminder, invitation cards and caregiver workshops can be used.

4) To offer pre-test to the caregiver during the first admission of the schizophrenia patient in the ward and post-test during discharge prior creating the discharge summary, this will enable to evaluate the knowledge and attitude of the caregiver of a schizophrenia patient. 


\section{Suggestions for Further Research}

1) A follow up study could be undertaken to evaluate the knowledge and attitude of the caregivers of schizophrenia patients who had been discharged from the facility.

2) A randomized trial to assess the effectiveness of education programs for caregivers of schizophrenia patients can be undertaken.

\section{References}

[1] APA. (2017). Supplement to Diagnostic and Statistical Manual of Mental Disorders, 5th edition. Washington DC: American Psychiatric Association Publishers.

[2] Barry, P. (2013). Mental Health and Mental Illness 7th edition. New York: Lippincott ravens Publishers.

[3] Townsend, M. (2013). Nursing Diagnoses in Psychiatric Nursing: Care Plans and Psychotropic Medications, 9th edition. Oklahoma: F. a Davis Company.

[4] Olfson, G. (2015). Premature Mortality Among Adults With Schizophrenia. London: JAMA Psychiatry.

[5] MOH. (2016). Kenya Mental Health Policy 2015-2030; Towards Attaining the Highest Standards of Mental Health. Nairobi: Ministry of Health.

[6] MOH Kenya. (2009). Clinical Guidelines for Diagnosis and Treatment of Common Conditions in Kenya. Nairobi: Ministry of Health Kenya.

[7] Ndetei, D. (2016). Schizophrenia and Other psychotic Disorders. Nairobi: University of Nairobi.

[8] Ferrari, C., \& Santomauro, D. (2018, may 12). Global Epidemiology and Burden of Schizophrenia. Retrieved from PubMed: https://www.ncbi.nlm.nih.gov/pubmed/29762765
[9] Gomez, D. (2015). Attitudes About Schizophrenia; Worlwide Campaing againist the Stigma of Schizophrenia. Social Psychiatry, 12-15.

[10] Chabungbam, G. (2014). Sociodemographic and clinical factors associated with relapse in Schizophrenia. Chandigarh: Folia publishing Society.

[11] NASCOP. (2018). Guidelines on Use of Antiretrovial Drugs for Treating and Preventing HIV in Kenya. Nairobi: NASCOP.

[12] Mbugua, J. M. (2016). Stigma Towards Mental Illness in a Rural Community in Kenya. Nairobi: Unpublished Academic, University of Nairobi.

[13] Igberase, O., \& Okogbenin, E. (2017). Beliefs About the Cause of Schizophrenia in Midwestern Nigeria. Benin: Delta State University.

[14] Magaru, M. (2012). Knowledge, Attitude and Practices of Caregivers of Patients with Schizophrenia in Port Moresby, Papua new guinea. Port Moresby: Pacific Journal of Medical Sciences.

[15] Gathaiya, N. W. (2013). Factors Associated with Relapse in patients with Schizophrenia at Mathari Teaching and Refferral Hospital. Nairobi: Unpublished Academic, University of Nairobi.

[16] Shinde, M. (2014). Knowledge, Attitude and Practices among Caregivers of Patients with Schizophrenia in Western Maharashtra.

[17] Oye, V. L. (2018, January 2). Community Study of Knowledge and Attitude to Mental Illness in Nigeria. Retrieved from The British Journal of Psychiatry: https://doi.og/10.1192/bjp.186.5.436 International Journal of Science and Research, 1-7. 